Dr. Ahmed Aboul Serour Lecturer in Ob/Gyn International Islamic Center for Population Studies and Research Al Azhar University
Corresponding Author: Ahmed Serour Lecturer in Ob/Gyn IICPSR- Al Azhar University Mobile: 0201223257918 Email: ahmedaboulserour@ yahoo.com Address: 5, Lebanon St., CairoEgypt

\begin{abstract}
Polycystic Ovarian Syndrome(PCOS) affected 5-15\% of women and is the commonest cause of hirsutism in women. The prevalence of hirsutism in PCOS patients is $70-80 \%$ compared with $4 \%-11 \%$ in women in the general population.

Methods: A search in the Medline, Pub Med, Cochrane library database and Embase was made. Prospective randomized trials, systematic reviews,meta-analysis and retrospective studies on hirsutism and PCOS were extracted and reviewed. Evidence for the different outcomes and recommendations for interventions were used on the best available data.
\end{abstract}

Review of Evidence: Evidence revealed that there is no universal agreement on the definition of PCOS. The NIH, ESHRE and ASRM and Androgen Excess and PCOS Society use different definitions based on the three criteria of Oligo anovulation (OA), biochemical or clinical hyperandrogenamia (HA) and polycystic ovarian morphology (PCOM). Moleular pathways modulating androgen biosynthesis and their relationship to insulin metabolism have enhanced our understanding of the pathophysiology of PCOS and its therapy. Hirsutism in PCOS is due to excess production of ovarian androgen driven by high level of LH.

Life style modification and loss of body weight in PCOS patient complaining of hirsutism should be advised. Effective medical treatment includes, combined hormonal contraceptives (CHCs), antiandrogens or combined CHCs and antiandrogens. Insulin sentizers are also useful when there is associated hyperinsulinaemia.

Short term and long term effective methods for treatment of hirsutism are also available and effective.

Conclusion: The strategy for management of PCOS patients with hirsutism should be patient centered. Medical treatment and cosmetic treatment are effective. Long term sequela of PCOS should not be overlooked..

Key words: PCOS, hirsutism, hyperandrogenamia (HA), antiandrogens, CHCs.

The association of polycystic ovary (PCO) and hirsutism dates back to 1935 when Irving Stein and Michael Leventhal reported a case series of seven women characterized by amenorrhea associated with hirsutism and bilateral PCO. ${ }^{(1)}$

Later on the term polycystic ovarian syndrome (PCOS) was developed, a condition that affects $5-15 \%$ of women ${ }^{(2)}$. It was realized that PCOS is a heterogeneous syndrome, not a disease. Patients with PCOS have a set of phenotypic characteristics but lack a single defining element or "gold standard"on which the diagnosis could be anchored. It is therefore not surprising that for a long time there was no agreement in the literature on the definition of PCOS. Three sets of criteria have been proposed. The National Institute of Health (NIH) criteria are (oligo-anovulation and biomedical or clinical huperandrogenism ${ }^{(3)}$. The Rotterdam consensus 
criteria was developed in 2003 by the European society of Human Reproduction and Embryology (ESHRE) and the American Society of Reproductive Medicine (ASRM) working group. The group agreed on a consensus for the definition of PCOS which has been extensively used in the literature and known as Rotterdam definition of ${ }^{(4)}$. The Rotterdam consensus defined PCOS as a syndrome characterized by the presence of at least two of three manifestations namely, Oligo and/or anovulation (OA), clinical/ or biochemical signs of hyperadrogenism (HA), and polycystic ovarian morphology (PCOM). PCOM was defined by the presence of either 12 or more follicles measuring 2-9 $\mathrm{mm}$ in diameter per whole ovary or increased ovarian volume $>10 \mathrm{~cm} 3$. When diagnosis of clinical and or biochemical signs of hyperandrogism is made one must excluded other etiologies such as congenital adrenal hyperplasia, androgen secreting tumorous, cuching syndrome and hyperprolactinaemia.

Based on the Rotterdam definition there are four phenotypes of PCOS as shown in table I:

Table 1: PCOS Phenotypes

\begin{tabular}{|c|c|c|c|}
\hline Phenotype & OA & HA & PCOM \\
\hline A & + & + & + \\
\hline B & + & + & - \\
\hline C & - & + & + \\
\hline D & + & - & + \\
\hline
\end{tabular}

In 2009, the Androgen Excess Society (AES) launched a new definition that considers PCOS primarily as a disorder of clinical and/or biochemical androgen excess, plus either chronic oligo-anovulation and/or polycystic ovary ${ }^{(5)}$. Some authors questioned whether it is appropriate to apply the definition of PCOS in the absence of overt $\mathrm{HA}^{(6)}$. Rotterdam consensus has been there for more than 13 years and a lot of developments has happened in the diagnostic tools of PCOS such as the presence of the latest generation of ultrasound machines, and the serum anti-Mullarian hormone $(\mathrm{AMH})$ assessmentto warrant rethinking of the criteria for diagnosis of PCOs ${ }^{(7)}$.

Pathophysiology of androgen biosynthesis in PCOS

\section{Hyperandrogenism in PCOS}

Androgens are produced primarily from dietary cholesterol that circulates in the form of low-density Lipoproteins (LDL) in the plasma ${ }^{(8)}$. In the steroid producing cells, LDL cholesterol can either be stored in lipid droplets for future use or converted into free cholesterol and utilized immediately for androgen biosynthesis ${ }^{(9)}$. Beside the adrenal glands and ovaries, peripheral tissues including adipose tissues, hair follicles and genital skin also contribute to androgen biosynthesis and circulating androgens ${ }^{(10)}$.

In women, androgens are secreted in almost equal quantities by the ovaries and adrenal glands ${ }^{(11)}$ and the enzymes involved in the steroidgenesis pathway are similar ${ }^{(12)}$. Thus both ovarian and adrenal-derived cell lines have been employed for basic research examining the regulation of steroidogenesis. They provided useful tools for studying drugs that may modulate androgen production.

The discovery of molecular pathways modulating androgen biosynthesis, and their relationship to insulin metabolism have enhanced our understanding of the pathophysiology of PCOS. Studies with ovarian theca cells taken from women with PCOS have demonstrated increased androgen production due to increased CYP17AI and HSD3B2 enzyme activities that produce androgens from cholesterol ${ }^{(13)}$. Furthermore increased expression of DENND1 A variant 2 was detected in the theca cells taken from women with PCOS. Moreover. over expression of DENN1A variant 2 in normal theca cells resulted in a PCOS phenotype with increased androgen production ${ }^{(14)}$. Recent breakthroughs have demonstrated that dietary medium - chain fatty acid decanoic acid can restore estrous cyclicity and lower androgen levels in an animal model of PCOS ${ }^{(14)}$. This discovery lays the platform for novel therapeutic development in PCOS.

In women, the major circulating androgens or proandrogens in a descending order of serum concentration are dehydroepiandrosterone sulphate (DHEAS), dehydroepiandrosterone (DHEA), androstenedione (A4), testosterone (T) and dihydrotestosterone (DHT). However, only $\mathrm{T}$ and DHT have strong affinity and potency for androgen receptors (AR). DHEAS, DHEA and A4 have little or no capacity to bind to the AR and require conversion to testosterone to exert androgenic effects. AT is the most important precursor of T while DHEAS and DHEA account for only 5\% and 13\% of circulating $\mathrm{T}$ among women of reproductive age respectively ${ }^{(15)}$.

In women the most common cause of hyperandrogenism is PCOS. Gonadotropin dependent functional ovarian androgen excess is the cause of this hyperandrogenemia. Ovarian theca cells increase androgen production in response to chronically elevated $\mathrm{LH}$ and insulin levels. Hyperinsulinemia promotes androgen biosynthesis via insulin receptor and to a lesser extent, the insulinlike growth factor-1 (IGF.1) receptor on theca cells and increases levels of circulating free testosterone 
by suppressing hepatic production of steroid hormone binding globulin $\mathrm{SHBG}^{(16,17)}$.

Testosterone is the most important circulating androgen because of its relatively high plasma concentration and greater potency at the target organ level. The ovaries and the adrenals account for $35-40 \%$ and $40 \%$ of the circulating testosterone respectively. The remaining circulating testosterone is derived from the conversion of androgenic precursors, mainly androstenedione, derived from the ovaries and adrenals, and dehydroepiandrosterone (DHEA), derived almost exclusively from the adrenals ${ }^{(18-20)}$.

Peripheral conversion of androgenic prohormones to testosterone occurs in the liver, skin and adipose tissue. In the circulation testosterone is present as the free or conjugated testosterone. Almost 98-99\% of plasma testosterone is bound to steroid hormonebinding globulin (SHBG), to cortisol-binding globulin or nonspecifically to albumin and other proteins and is biologically inactive. The free portion of testosterone is bidologically active.

In the hair follicles, testosterone is converted to its biologically active form, dihydrotestosterone, by the enzyme. $5 \alpha$ - reductase ${ }^{(18-20)}$.

Sex hormones work independently on the liver and control SHBG production. Estrogens increase and adnrogens decrease the production of SHBG in the liver. In hyperandrogenic condition, the SHBG is decreased thus allowing higher levels of free testosterone.

Insulin is another modulator of SHBG. Insulin decreases the production of SHBG. In condition of insulin resistance and compensatory hyperinsulinemia, the production of SHBG is decreased and this leads to higher levels of free testosterone.

\section{Pathogenesis of Hirsutism}

Androgens are produced primarily from dietary It is important to differentiate between hirsutism due to hyperandrogenism and excessive hair growth due to genetic or ethnic causes. The human body is covered by approximately 50 million hair follicles which cover every surface of the body except the palms and the soles of the feet ${ }^{(21)}$. The number of hair follicles does not increase after birth but slowly begins to decrease at around age of forty. The density and pattern of hair follicles vary by the ethnic origin and genetics. Women of Mediterranean origin generally have much more hair than Asians and American Indians.

Adults have two types of hair, vellus and terminal. Vellus hair is soft, fine, generally colorless, and usually short. Terminal hair is long, coarse, dark, and sometimes curly. In most women, vellus hair covers the face, chest, and back and gives the impression of "hairless" skin while in most men, terminal hair covers the face and body. Terminal hair grows on the scalp, pubic, and armpit areas in both men and women. A mixture of vellus and terminal hair covers the lower arms and legs in both men and women. Hirsutism due to hormonal causes is the excessive growth of coarse dark hair on the face, chest, abdomen, back upper arms or upper legs of women ${ }^{(21)}$. Excessive hair that is due to genetic and ethnic variation rather than hormonal causes is typically located on the arms, hands, legs, and feet. If excessive hair growth is present only on the lower legs and forearms, it is not considered hirsutism and will not respond to hormonal therapy.Hair growth occurs in cycles. While some hair follicles grow, others rest, and still others are shed.If hair follicles are hormone-sensitive, androgens may cause some vellus hairs to change to terminal hairs and cause the terminal hairs to grow faster and thicker

\section{Hirsutism in PCOS}

Understanding the pathophysiology of hirsutism in PCOS as well as its severity and the distress it causes to each patient is essential to choose the proper treatment.

While androgen excess in PCOS patients will contribute to the ovulatory and menstrual dysfunction of PCOS patients the most recognizable sign of hyperadnrogenaemia includes hirsutism, an acne and androgenic alopecia or female pattern hair loss.

Hirsutism can arise from increased androgen production or from increased sensitivity of hair follicles to circulating androgens. Hirsutism is a sign, not a disease of itself. PCOS is the most common etiology of hirsutism. PCOS is found in $72 \%$ to $82 \%$ of patients with hyperandrogenism ${ }^{(20)}$.

The prevalence of hirsutism in PCOS ranges from $70-80 \%$ versus $4 \%$ to $11 \%$ in women in the general population ${ }^{(22)}$. Hirsutism in PCOS is associated with both ovarian derived androgen excess and individual sensitivity of the pilosebaceous unit to androgens.

The pilosebaceous unit (PSU) consists of a pilary component and sebaceous component. Each PSU has the capacity to form either a terminal hair (dark, pigmented, large modulated hair) or a sebaceous follicle in which the hair remains villous and the sebaceous gland is more prominent. Androgens control the development of PSU. When androgens increase PSUs from terminal hairs in sexual areas and increase the size of sebaceous glands in sebaceous areas. Excessive 
terminal hairs growth results in hirsutism. Excessive secretion from the sebaceous glands results in acne ${ }^{(23)}$.

Development of hirsutism is determined by androgen levels and the sensitivity of PSU to androgen. Increased sensitivity of PSU is caused by exaggerated peripheral $5 \alpha$ - reductase activity, androgen receptor polymorphism, or altered androgen metabolism(19). Thus, the level of androgens does not always correlate to the degree of hirsutism.

\section{Other causes of Hirsutism}

While PCOS and idiopathic causes account for almost $90 \%$ of cases of hirsutism ${ }^{(19,20)}$ there are other rare causes of hirsutism.In older women ovarian secretion declines rapidly and ovarian androgen production continues for a few years after menopause. This gonadotrophic dependent androgen when excessive can lead to hirsutism ${ }^{(24,25)}$. Ovarian tumours and hyperthcosis producing androgens can cause hirsutism. There are several non ovarian causes of hirsutism ${ }^{(20,26)}$.

Adrenal causes include Cushing's syndrome, androgenproducing tumors and congenital adrenal hyperplasia $(\mathrm{CAH})$ resulting from 21 hydroxylase deficiency, $11 \beta$ hydroxylase deficiency and $3 \beta$ hydroxysteroid dehydrogenase deficiency.

Hydperprolactinaemia stimulates adrenal DHEA sulphate (DHEAS) production, causing hirsutism. Hypothyrodism can also cause hirsutism.

Exogenous drugs can cause hirsutism. These include Valproic acid, a commonly used antiepileptic agent (27), anabolic steroids used for increase in muscle mass in chronically ill and debilitated people and some bodybuilding's supplements such as DHEA and A4 (21). Severe insulin resistance syndrome such as maturityonsetdiabetes of the young and lipodystrophies are rare causes of hirsutism ${ }^{(28)}$.

Some women are born with insulin resistance, a defect in the ability of insulin to control blood sugar levels. The pancreas to compensate for this insulin dysfunction,secretes more insulin. High levels of insulin stimulate the ovaries to overproduce androgens leading to hirsutism, acne, and irregular ovulation. Insulin resistance may lead to diabetes mellitus, hypertension, heart disease and excessive growth and darkening of the skin a condition known as acanthosis nigricans (Hair-AN syndrome) which usually occurs around the crease areas of the skin and the neck ${ }^{(21)}$. The majority of these cases are genetic in origin. It should not be confused with the milder insulin resistance seen in PCOS.

\section{Diagnosis:}

\section{A-Clinical assessment:}

The Health Care Providers (HCPs) should make the distinction between terminal hairs growing in a male pattern indicating hirsutism and hair growth due to genetic or ethnic predisposition. He/She should enquire of history of intake of androgenic drugs.

HCPs should differentiate between hirsutism due to PCOS or other previous listed causes using clinical, biomedical, pelvic sonography or special X-rays if necessary. The goals of biochemical assessment are to evaluate the source of hyperandrogenism and to exclude the presence of malignancy.

\section{B-Hormaonal assessment:}

\section{1- Testosterone assessment:}

Testosterone total and free should be measured in early morning samples. The testosterone levels are usually high-normal or just at the upper limit of normal Values of testosterone higher than $200 \mathrm{ng} / \mathrm{mL}$ suggest an androgen producing tumour ${ }^{(28)}$. Assessment of testosterone requires the use of the highest quality assays available, generally radioimmunoassay with extraction and chromatography or mass spectrometry preceded by liquid or gas chromatography.

\section{2- DHEAS assessment:}

Marginal elevation of DHEAS are common but elevated values suggest an adrenal source of androgens. Levels higher than $700 \mu \mathrm{g} / \mathrm{dL}$ suggest an androgen producing adrenal tumour ${ }^{(28)}$.

\section{3- 17 Hydroxyprogesterone assessment:}

17 ydroxyprogesterone assessment in a morning sample is a screening test for adult onset CAH. Levels lower than $200 \mu \mathrm{g} / \mathrm{dL}$ exclude the disease. Mildly increased levels between 300 to $1000 \mu \mathrm{g} / \mathrm{dL}$ require ACTH stimulation test. Post stimulation values above $1000 \mu \mathrm{g} / \mathrm{dL}$ indicate a positive result.

\section{4- Free cortisol:}

Free cortisol in a 24- hour urine sample should be measured if Cushing's syndrome is suspected.Prolactin levels are mildly elevated in about $20 \%$ of patients with PCOS.

\section{5- Serum LH assessment:}

Serum LH should be measured. It is elevated in women with PCOS and a ratio of LH/FSH levels higher than 3 is significant ${ }^{(28)}$. 


\section{C-Ultrasound evaluation:}

Ultrasound evaluation of the ovaries, adrenals or both may be useful forsscreening if symptoms or biochemical levels suggest the presence of neoplasm.

The HCPs should not ignore the fact that the diagnostic label of PCOS implies an increased risk for infertility, dysfunctional uterine bleeding, endometrial cancer, obesity, type II diabetes, dyslipidemia, hypertension and possibly cardiovascular disease ${ }^{(29)}$. The diagnostic accuracy of PCOS as the cause of hirsutism is therefore clinically, socially and financially important particularly in adolescents.

\section{Evaluation of Hirsutism}

Hirsutism is evaluated by scoring facial and body terminal hair growth using the modified FerrimanGallwey method and recording and scoring of acne and alopecia. However, the hirsutism score correlates poorly with serum androgens ${ }^{(30)}$. Many authors consider that the sole complaint of "unwanted hair growth" in the absence of frank hirsutism on physical examination, may signal the presence of PCOS.It is not the same for acne especially among adolescent.Alopecia must meet the definition of androgenic alopecia ${ }^{(7)}$.

\section{Management of Hirsutism in PCOS}

The patient needs to be assessed and evaluated. Treatment should be patient centered whether for hirsutism only or hirsutism and anovulation, anovulatory bleeding or desire to get pregnant.

Initiation of treatment should be based on the patient's perception of hirsutism rather than the quantitative characteristics of hirsutism ${ }^{(31)}$.

The management of hirsutism due to PCOS involves primarily either androgen suppression, with a hormonal combination contraceptive or androgen blockade as with androgen receptor blocker or a $5 \alpha$ reductase inhibitor or a combination of the above $\mathrm{e}^{(2)}$.

Medical treatment should be combined with cosmetic treatment including the use topical eflornithine hydrochloride and short term (shaving, chemical depilation, plucking, threading, waxing and bleaching) and long-term (electrolysis, laser therapy and intense pulse light therapy) mechanical treatments ${ }^{(2)}$.

Furthermore monitoring of $\mathrm{T}$ and other androgens during treatment is generally unnecessary as the hirsutism score correlates poorly with serum androgens ${ }^{(32)}$.

Smoking cessation is strongly recommended for hirsute patients as many of the undesirable side effects of the medications prescribed to treat hirsutism are exacerbated when patients indulge in smoking ${ }^{(33)}$.

Generally acne responds to therapy relatively early while hirsutism is slower to respond with improvements observed as early as three months, but generally only after 6-8 months of therapy ${ }^{(2)}$.

\section{Hyperandrogenism in PCOS}

Androgens are produced primarily from dietary cholesterol that circulates in the form of low-density Lipoproteins (LDL) in the plasma ${ }^{(8)}$. In the steroid producing cells, LDL cholesterol can either be stored in lipid droplets for future use or converted into free cholesterol and utilized immediately for androgen biosynthesis ${ }^{(9)}$. Beside the adrenal glands and ovaries, peripheral tissues including adipose tissues, hair follicles and genital skin also contribute to androgen biosynthesis and circulating androgens ${ }^{(10)}$.

In women, androgens are secreted in almost equal quantities by the ovaries and adre

\section{Hormonal Combination contraceptives HCCs}

Progestins in HCCs causes suppression of LH levels and inhibition of LH-mediated ovarian androgen synthesis ${ }^{(34)}$ Ethinylestrodial in HCCs leads to significant increase in SHBG, thereby contributing to a reduction of free $\mathrm{T}^{\left({ }^{(3)}\right)}$. HCCs decrease the synthesis and release of androgens by the adrenal(36).60-100\% of women with hirsutism demonstrate improvement on oral HCCs ${ }^{(37)}$. Twenty one day active /17 day placebo provides better ovarian suppression compared to continuous regimen ${ }^{(38)}$.

Some HCCs have progestins with antiandrogenic properties such as Cyproterone acetate (CPA), Chlormadinone acetate (CMA), Drospirenone (DRSP), DIENOGEST (DNG). These progestins are available in combination with estrogens such as ethinyl estradiol or estradiol valerate. Comparative studies of HCCs containing progestin with antiandrogenic properties are limited ${ }^{(39)}$. A randomized controlled trial demonstrated that HCCs with DRSP were more effective in the reduction of clinical hyperandrogenism as compared with CMA- containing HCCs ${ }^{(40)}$. In a recent RCT it was demonstrated that after 12 months of treatment, use of HCCs containing CPA was associated with a significant decrease in the $\mathrm{mFG}$ score as compared with HCCs containing both desogestral (DSG) and drospirenone (DRSP) ${ }^{(41)}$. 


\section{$\underline{\text { GnRHa and Insulin Sensitizers }}$}

Several studies have demonstrated the superior efficiency of HCCs monotherapy compared to $\mathrm{GnRHa}^{(42)}$ and insulin sensitizers ${ }^{(43)}$.

\section{Antiandrogens Monotherapy}

The antiandrogens commonly used in hirsutism due to PCOS include androgen receptor (AR) blockers, Spironolactone, Flutamide, Cyproterone acetate, and $5 \alpha$ reductase inhibitor. Antiandrogens have not been used with any regularity in women.All are teratogenic and their use is generally discouraged ${ }^{(39)}$. Four RCTs demonstrated that antiandrogens in combination with HCCs were more effective than monotherapy with $\mathrm{HCCs}^{(44)}$.

Because efficacy is generally higher when using a combination of HCCs and antiandrogens than with either HCC or antiandrogen monotherapy, and because HCCs minimize the risk of teratogenicity it is advisable to begin therapy with a combination of HCCs and antiandrogens ${ }^{(39)}$.

\section{Combined treatment of antiandrogen with metformin}

In a small RCT, flutamide in combination with metformin appeared to be superior to monotherapy ${ }^{(45)}$.

A systematic review of RCTs has shown that the effect of treatment was negatively associated with BM/ in PCOS ${ }^{(45)}$. A systematic review of six small RCTs observed that lifestyle modification was beneficial in the reduction of serum androgens and increased SHBG, along with some improvement in hirsutism as achieved by the mFG Score ${ }^{(46)}$.

\section{Cosmotic approaches}

Cosmotic approaches for treatment of hirsutism are widely used and categorized as short and long term approaches.

\section{$\underline{\text { Short term Methods }}$}

These include shaving, chemical, depilation, plucking (threading), waxing, bleaching, and eflornithine hydrochloride.

Depilation is the removal of the hair shaft from skin's surface as shaving and chemical depilation. Epilation is the extraction of hair above the bulb (eg plucking, waxing). It provides the most long lasting action on hair re-growth with hair absent for 6-8 weeks ${ }^{(47)}$.

Topical application of $13.9 \%$ eflornithine hydrochloride for facial hirsutism inhibits the enzyme ornithine decarboxylase which is required for the growth and differentiation of cells in the hair follicle. It requires daily use and hirsutism relapses after 8 weeks of cessation of treatment, and is not approved for large surface $\operatorname{area}^{(48)}$.

These short term methods are relatively safe and affordable, side effects as dermatitis, bleaching and skin irritation are rare.

\section{Long Term Methods}

These include, Electrolysis, Laser therapy, and Intense pulsed light (IPL) ${ }^{(49)}$.

Electrolysis has been commonly used for treatment of hirsutism since $1875^{(50)}$.

Electrolysis facilitates chemical destruction of the dermal papilla. Shaving few days before electrolysis is helpful as it allows selective destruction of growing anagen hair ${ }^{(51)}$. Laser therapy is based on selective photothermolysis. The melanin of the hair follicle accumulates the light energy which destroys the hair bulb ${ }^{(52)}$. Several lasers with varying wavelength are available. Barker skin are usually more difficult to treat using laser as light energy is absorbed by the surrounding epidermis of the hair follicle. A Cochrane review showed $50 \%$ hair reduction in a period of 6 months after treatment ${ }^{(53)}$.

Intense pulsed light (IPL) is superior to the ruby laser, similar to the Nd: YAG laser and less effective than diode laser therapy ${ }^{(54)}$. Data regarding efficacy of IPL are rather limited ${ }^{(55)}$. It is associated with less risk of burning in patients with darker skin.

\section{Conclusion}

PCOS is a heterogenous syndrome which affects $5-15 \%$ of women and is the commonest cause of hirsutism in women. Patients with PCOS have a set of phenotypic characteristics but lack a single defining element of "gold standard" on which diagnosis could be anchored.

Hyperandrogenemia is an important feature of PCOS and it causes hirsutism, acne and loss of female pattern hair distribution. In PCOS excess androgen production is driven by a high level of $\mathrm{LH}$.

It is important to diagnose the cause of hirsutism before starting treatment. The treatment of hirsutism due to PCOS should be patient centered and takes 
into consideration other associated complaint of the patient such as infertility and the long term sequaelae of PCOS.

Medical treatment of hirsutism includes combined Hormonal Contraceptive, antiandrogens, combined hormonal contraceptives and antiandrogens and insulin sentizers.

Changing life style, reducing body weight and giving up smoking improve response to medical treatment. Long term and short term cosmotic therapy may be need in addition to medical treatment.

\section{References}

1. Stein IF, Leventhal ML, 1935. Am. J Obstet / Gynecol, 29:181-91.

2. Yong EL, 2016. Polycystic ovarian syndrome. Clinical Obstetrics and Gynecology. 37 (2016) 1-4.

3. Zawadzkik and Dunaif A, 1995. Diagnostic criteria for polycystic ovary syndrome towards a rational approach. In Dunaif A (ed) Polycystic Ovary Syndrome. Boston: Blackwell Scientific, 377-384

4. Rotterdam ESHRE/ASRM- sponsored PCOS consensus workshop group. Revised 2003 consensus on diagnostic criteria and long term health risks related to PCOS. Hum. Reprod. 2004: 19 (1): 41-7.

5. Azziz R, Carmina E, Dewailly D et al. Task Force on phenotype of the PCOS of the androgen Excess and PCOS Society. The androgen Excess and PCOS society criteria for the PCOS. The complete task force report. Fertil and Steril 2009: 91,456-488.

6. DewaillyD,Catteau- Jonard S, REyss AC et al 2006. Oligo anovulation with PCO but not overt HA. J clin endocrinal Metab. 91 (10): 3922-7.

7. Dewailly D 2016. Diagnostic criteria for PCOs:Is there a need for a rethink? Best practice and Research clinical obstetrics and gynecology, 37, (2016), 5-11.

8. Gwynne JT, Strauss JF. The role of lipoproteins in steroidogenesis and cholesterol metabolism in steroidogenic glands. Endocr Rev. 1982:3:299-329.

9. Kramer FB Adrenal cholesterol utilization. Mol cell endocrinol 2007; 265-266: 42-5.

10. Papadopoulos V, Vihma V. Steroid biosynthesis in adipose tissue. Steroids 2015; 103: 89-104.

11. Carmina E (2006). Ovarian and adrenal hyperandrogenism Ann NY Acad Soc. 2006: 1092:130-7.

12. Miller WL, Aushus RJ. (2011). The molecular biology, biochemistry and physiology of human steriodogenesis and its disorders. End. Rev 2011; 32:81-151.

13. Yong EL, Baired DT, Hillier SG. Mediation of gonadotrophin-stimulated growth and differentiation of human granulosa cells by adenosine-3 5monophospated: one molecule, two messages. Clin. Endocrinol. 1992:37:51-58.

14. Indran I R et al 2016. Cellular and animal studies: Insights into pathophysiology and therapy of PCOs. Best practice and Research clinical Obstet and Gynecol 37 (2016) 12-14.

15. Longcape C. Adrenal and Gonadol androgen secretion in normal females. Clin. EndocrinolMetab. (1986): 15 (2) 213-228.

16. Landy M , Huang A, Azziz R. Degree of hyperinsulinemia, independent of androgen levels, is an important determinant of the severity of hirsutism in PCOs. Fertil and Steril, 2009; 92 (2): 643-7.

17. Ovalle F, Azziz R. Insulin resistance, PCOs and type 2 diabetes mellitus. Fertil\&Steril 2002; 77 (6): 1095-105.

18. Depleski D, Rosenfield RL: Role of hormones in pilosebaceous unit development [review]. Endocr Rev 2000; 21:363-392.

19. Azziz R, Carmina E, Sawaya ME: Idiopathic hirsutism [review]. Endocr Rev 2000; 21:347-362.

20. Azziz R, Sanchez LA, Knochenhauer ES et al 2004. Androgen excess in women: experience with over 1000 consecutive patients. J Clin Endocrinol Metab: 89 (2): 453-62.

21. American society of Reproductive Medicines (ASRM) 2016. Hirsutism and PCOS. A guide for patients, 2016.

22. SpritzerPM, BaroneCR, OliveriaFB2016. Hirsutism in PCOS. Pathophysiology and management.

23. Kiddy DS, Hamilton-Fairley D, Bush A et al 1992. Improvement in endocrine and ovarian function during diatary treatment of obese women with PCOS. Clin Endcrinol (Oxf.) 1992; 36:105-111.

24. Lindgren R, Gunnarsson C, Jakobson A, Hammar $\mathrm{M}$ : Hyper secretion of ovarian androgens may be gonadotrophin-dependent years after menopause. Maturitas 2000; 34:43-46.

25. Barth JH, Jenkins M, Belchetz PE: Ovarian hyperthecosis, diabetes and hirsuties in postmenopausal women. Clin Endocrinol (Oxf) 1997; 46:123-128.

26. Legro RS, Aslanian SA, Ehrmann DA et al. Diagnosis and treatment of PCOS: an Endocrine Society clinical Practice guideline. J Clin. Endocrinol Metab. 2013:98(12):4565-92.

27. Genton P, Bauer J, Duncan S, et al: On the association between valproate and polycystic ovary syndrome [review]. Epilepsia 2001; 42:295-304.

28. Chinnappo P and Mehta A (2010): Hirsutism. Cleveland clinics. Center for continuing education. 
29. Hickey M, Doherty DA, Atkinson H et al 2011. Clinical, ultrasound and biochemical features of PCOS in adolescents implications for diagnosis. Human Reprod Vol 26, No. 6 PP 1469-1477, 2011.

30. Haung A, Landay M; Azziz R. The association of androgen levels with the severity of hirsutism in the PCOS. Fertil \& Steril: 86 S12-S, 2006.

31. Martin KA et al 2008. Evaluation and treatment of hirsutism in pre menopausal women: an endocrine society clinical practice guideline. J. Clin Endocrinol Metab. 93(4) 1105-20.

32. Legro RS, Schlaff WD, Diamond MP et al 2010. Total testosterone assays in women with PCOS precision and correlation with hirsutism. J Clin Endocrinol Metab. 95(12) 5305-13.

33. ESCobar-Morreale HF, Carmino E. Dewaill D, et al 2012. Epidemiology, diagnosis and management of hirsutism: a consensus statement by the Androgen Excess and PCOS society. Hum. Reprod. Update. 18(2): 146-70.

34. Archer DF, Kovalevsky G, Ballagh SA, et al. Ovarian activity and safety of a novel lovonorgestrel/ ethinylestradiol continuous oral contraceptive regimen. Contraception 2009; 80 (3): 245-53.

35. Vrbikova J, Cibula D. Combined oral contraceptives in the treatment of polycystic ovary syndrome. Hum. Reprod Update 2005; 11(3): 277-91.

36. Madden JD, Milewich I, Parker Jr CR,et al. The effect of oral contraceptive treatment on the serum concentration of dehydroisoandrosterone sulfate. Am. J Obstet Gynecol1978; 132(4): 380-4.

37. Burkman JR RT. The role of oral contraceptives in the treatment of hperandrogenic disorders. Am. J M Med 1995; 98(1A) 130S -6S.

38. Legro RS, Pauli JG, Kunselman AR et al. Effect of continuous versus cyclical oral contraception: a randomized controlled trial. J. Clinical Endocrinol Metab 2008; 93 (2): 420-9.

39. Lizneva D, Gavrilova- Jordan L, Walker W et al 2016. Androgen excess: Investigations and management. Best Practice and Research Clinical Obstet and gynecol 37:98-118.

40. Lello S, Primavera G, Clonna L et al. Effects of two estrprogenstins containing ethinylestradiol 30 microg and drospirenone $3 \mathrm{mg}$ and ethinylestradiol 30 microg and chlormadinone $2 \mathrm{mg}$ on skin and hormonal hyperandrogenic manifestations. Gynecol Endocrinol 2008; 24(12):718-23.

41. Bhattacharya SM, Jha A. Comparative study of the therapeutic effects of oral contraceptive pills containing desogestreal, cyproterone acetate, and drospirenone in patients with PCOS. Ferti Steril 2012, 98(4):1503-9.
42. Heiner JS, Greendale GA, Kawakami AK, et al. Comparison of a gonadotropin-releasing hormone agonist and a low dose oral contraceptive given alone or together in the treatment of hirsutism. J Clin Endocrinol Metab1995; 80(12) 3412-8.

43. Costello M, Shrestha B, Eden J. et al.Insulin sensitising drugs versus he combined contraceptive poll for hirsutism acne and risk of diabetes, cardiovascular disease, and endometrial cancer in polycystic ovary syndrome. Cochrane Database Syst Rev 2007 (1) CD 005552.

44. Swiglo BA, Cosma M, Flynn DN, et al. Clinical review: Antiandrogens for the treatment of hirsutism: a systematic review and metaanalyses of randomized controlled trials. J Clin Endocrinol Metab 2008; 93 (4): 1153-60.

45. Koulouri O, Conway GS. A systematic review of commonly used medical treatments for hirsutism in women. Clin Endocrinol (Oxf) 2008;68 (5): 800-5.

46. Moran LJ, Hutchison SK, Norman RJ, et al.Lifestyle changes in women with polycystic ovary syndrome. Cochrane Database Syst Rev 2011; (7) CD007506.

47. Ramos-e-Silva M, de Castro MC, Carneiro Jr LV. Hair removal. Clin Dermatol 2001;19 (4) 437-44.

48. Wolf Jr JE, Shander D, Huber F,et al. Randomized, double -blind clinical evaluation of the efficacy and safety of topical eflornithine $\mathrm{HCl} 13.9 \%$ cream in the treatment of women with facial hair. Int J Dermatol 2007;46(1): 94-8.

49. Martin KAChang RJ, Ehrmann DA, et al.Evaluation and treatment of hirsutism in premenopausal women: An Endocrine Society Clinical Practice Guideline J. Clin Endocrinol Metab2008; 93(4): 1105-20.

50. Richard RN, Maharg GE. Electolysis observations from 13 years and 140000 hours of experience. Am Acad Dermatol. 1995;33(4):662-6.

51. Urshibata O, Kase K, A comparative study of axillar hair removal in women: plucking versus the blend method. J. Dermatol 1995; 22(10): 738-42.

52. Liew SH. Laser hair removal: guidelines for management. Am J. Clin. Dermatol, 2002.

53. Haeders dal M, Gotzsche PC. Laser and photepilation of unwanted hair growth. Cochrane Database Syst Rev. 2006; (4) ICD004684.

54. Klein A, Seinert S, Beaumler W et al. Photoepilation with a diode laser versus intense pulse light: a randomized intrapatient left to right trial. Br. J Derm 2013; 168(6): 1287-93.

55. Headresal M, Wolf HC. Evidence-based review of hair removal using laser and light sorces. J. Eur Acad Dermatol venereal 2006; 20(1): 9-20. 